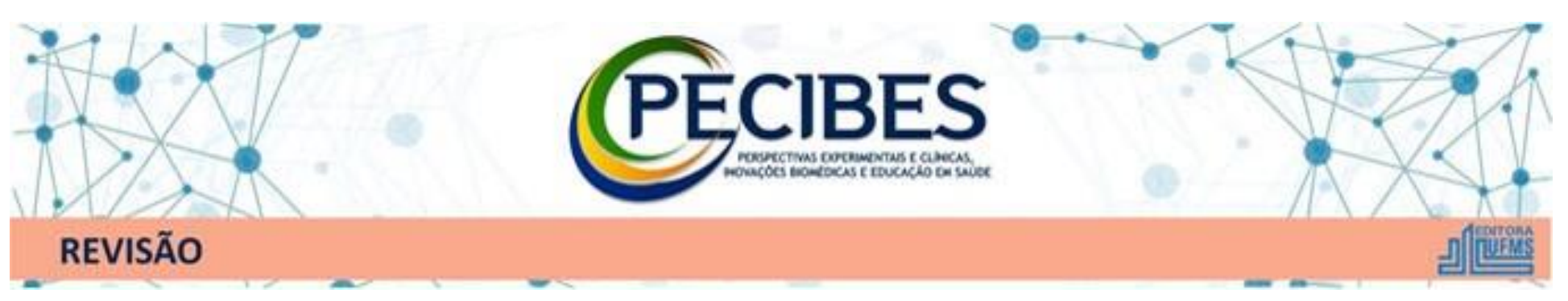

\title{
Lesões Endoperiodontais: classificação e diagnóstico
}

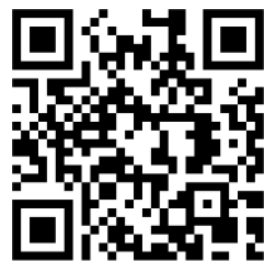

http://www.seer.ufm s.br/index.php/pecib es/index

*Autor correspondente: Emilly Cristina Costa Borges E-mail do autor: emillyborgesodo nto@gmail.com

Palavras-chave: Lesões endoperiodonta is. Polpa. Periodonto. Diagnóstico. Tratamento.

Key-words:

Endo-

periodontal

lesions. Pulp.

Periodonto.

Diagnosis.

Treatment.
Endoperiodontal Lesions: Classification and Diagnosis

Emilly Cristina Costa Borges ${ }^{1}$, Luiz Fernando Moreira Maziero ${ }^{2}$

${ }^{1}$ Acadêmica do Curso de Odontologia da UNIDERP.

${ }^{2}$ Mestre em Dentística Restauradora pela Universidade do Sagrado Coração e Especialista em Endodontia pela $\mathrm{ABO} / \mathrm{MS}$.

\section{Resumo}

As lesões endoperiodontais são consideradas complexas devido à inter-relação polpa e periodonto, o que exige do cirurgião-dentista não só o conhecimento básico da anatomia dental e das doenças endodônticas e periodontais, mas sim a compreensão da correlação de ambos, suas classificações, particularidades e as técnicas para um bom diagnóstico. O objetivo do trabalho é demonstrar essa complexidade de diagnóstico das lesões endoperio, bem como suas classificações. Diversos autores apresentaram classificações com intuito de auxiliar no diagnóstico preciso, junto da variedade de técnicas odontológicas como por exemplo: exame radiográfico, exame visual, sondagem periodontal, testes de vitalidade pulpar, palpação, percussão e mobilidade, rastreamento de fístulas, exposição cirúrgica, visto que apenas um teste não é satisfatório para se obter resultados conclusivos. Utilizando-se das diversas ferramentas disponíveis para correta diferenciação, associada a observação detalhada das particularidades do caso em questão, o nível de conhecimento teórico e vivência do profissional, obtém-se por consequência, a correta seleção da sequência terapêutica. Embora a patogênese variada e a amplitude das técnicas de diagnóstico apresentarem desafios para o cirurgião-dentista, o tratamento das lesões endoperiodontais pode ser bem-sucedido desde que haja a completa compreensão e o conhecimento científico para o estabelecimento de um correto plano de tratamento. A longo prazo, observa-se que o prognóstico depende não só do tratamento correto a partir de um bom diagnóstico, mas também do acompanhamento com motivação ao paciente e da gravidade e extensão da lesão inicial.

\section{Abstract}

Endoperiodontal lesions are considered complex due to the interplay between pulp and periodontium, which requires the dentist not only the basic knowledge of dental anatomy and endodontic and periodontal diseases, but also the understanding of the correlation of both, their classifications, particularities and techniques for a good diagnosis. The objective of the work is to demonstrate this complexity of diagnosis of endoperative lesions, as well as their classifications. Several authors have presented classifications in order to assist in the precise diagnosis, along with the variety of dental techniques such as: radiographic examination, visual examination, periodontal sounding, pulp vitality tests, palpation, percussion and mobility, tracking of fistulas, surgical exposure, visa that only one test is not satisfactory to obtain conclusive results. Using the various tools available for correct differentiation, associated with detailed observation of the particularities of the case in question, the level of theoretical knowledge and experience of the professional, it is consequently obtained, the correct selection of the therapeutic sequence. Although the varied pathogenesis and breadth of diagnostic techniques present challenges for the dental surgeon, the treatment of endoperiodontal lesions can be successful as long as there is complete understanding and scientific knowledge for the establishment of a correct treatment plan. In the long term, it is observed that the prognosis depends not only on the correct treatment based on a good diagnosis, but also on motivated monitoring of the patient and on the severity and extent of the initial injury. 
As disfunções nos tecidos pulpares e periapicais são responsáveis por mais da metade das perdas dentais, por conseguinte, quanto maior o conhecimento a respeito desses tecidos, melhor a qualidade do tratamento odontológico e prognóstico que o cirurgião-dentista pode oferecer ao paciente. Com essa visão, as lesões endoperiodontais abrangem alta complexidade devido a inter-relação polpa e periodonto sendo assim primordial o diagnóstico diferencial.

O cirurgião-dentista precisa ter além do conhecimento básico da anatomia dental e das doenças endodônticas e periodontais, a compreensão da inter-relação polpa e periodonto, suas classificações, particularidades e as técnicas para um bom diagnóstico. Portanto, este projeto de pesquisa justifica-se pela relevância do tema identificado durante o levantamento de artigos, a fim de oferecer aos acadêmicos e profissionais da odontologia maior conhecimento sobre o assunto que é de alta complexidade e assim direcionar o correto diagnóstico para o melhor prognóstico possível para o paciente sobre tratamento odontológico.

Considerando a relevância do assunto proposto, com base na justificativa apresentada no parágrafo anterior e a fim de estimular a curiosidade e consequentemente a pesquisa sobre o relevante tema, pergunta-se quais as dificuldades no correto diagnóstico das complexas lesões endoperiodontais?

Este estudo objetivou demonstrar a complexidade de diagnóstico das lesões endoperiodontais bem como suas classificações, e especificamente apresentar o conceito das lesões endoperiodontais e suas classificações, descrever técnicas de diagnóstico das lesões endoperiodontais e elencar a importância do correto diagnóstico para o tratamento adequado das lesões endoperiodontais.

\section{Material e Métodos}

A pesquisa bibliográfica foi realizada nas bases de dados Scielo, Medline e Google Acadêmico de estudos clínicos, básicos e de revisão publicados em revistas com reconhecido impacto no meio acadêmico e científico. A pesquisa restringiu-se a trabalhos publicados com a busca de palavras-chaves: "Lesões endoperiodontais", "Polpa" e "Periodonto".

\section{Resultados}

O termo lesão endoperiodontal (LEP) ou lesão endoperio vem sido utilizado nos últimos anos para descrever os processos inflamatórios que não se encaixam apenas nas classificações de lesões endodônticas ou periodontais, e sim em ambas, pois são encontrados nos dois tecidos em diferentes níveis (CASTRO et al, 2011). As principais estruturas do periodonto são consideradas separadas do tecido dental apenas para fins didáticos, uma vez que além da intima relação física entre essas estruturas, estudos mostram vínculos de desenvolvimento embrionário entre polpa e periodonto (SANTOS; LINS, 2007).

Produtos tóxicos metabólicos de origem endodôntica ou periodontal podem fluir para as duas direções com facilidade devido a anatomia dental composta por vias de comunicação que ligam intimamente o periodonto e a polpa, sendo três principais: túbulos dentinários, canais laterais e acessórios e o forame apical (PENONI; ANDRADE, 2016). As vias de disseminação podem levar bactérias do tecido pulpar pelo interior do ligamento periodontal, causando uma periodontite retrógrada, ou a infecção pode coexistir em ambos os tecidos com a presença de colônias anaeróbias mistas (SHENOY; SHENOY, 2010).

Essas vias, em sua maior parte, são de origem anatômica, porém de acordo com Parolia et al (2013) os patógenos podem fluir entre os dois tecidos de forma patológica, através de fraturas radiculares por traumas, perda de cemento por irritantes externos, reabsorções da raiz por agentes internos ou externos e espaços vazios radiculares ocasionados pelas fibras de Sharpey, tal como de forma iatrogênica, através de procedimentos endodônticos mal planejados e/ou executados onde ocorrem perfurações ou fraturas radiculares e erros em procedimentos periodontais que geram exposições de túbulos dentinários.

Segundo Castro et al (2011) a complexidade de diagnóstico das lesões endoperio se dá pela variedade em sua origem, podendo ser a partir de uma destruição periodontal que se junta a uma lesão endodôntica ou uma doença pulpar que afeta na destruição do periodonto através de suas toxinas e materiais patógenos. Por tanto, para um bom diagnóstico é indispensável levar em consideração a história clínica do paciente e realizar exames radiográficos complementares, além da averiguação etiológica específica antes de optar por qualquer tipo de tratamento (FAGUNDES et al, 2007).

Ainda de acordo com Fagundes et al (2007), os microrganismos são os fatores etiológicos mais recorrentes dentro das lesões endoperio, destacando-se as bactérias anaeróbicas. Bactérias dos gêneros Peptostreptococcus, Eubacterium, Fusobacterium, Bacteroides e Streptococcus anaeróbios são encontradas tanto em polpa quanto no periodonto em diversos estudos de observação de amostras dentais. Entretanto, nem sempre as espécies que predominam no tecido periodontal são as mesmas do canal, fato que pode ser esclarecido pela diferença entre os nichos, sendo a microbiota endodôntica menos complexa do que a periodontal.

Com o propósito de diminuir a dificuldade de diagnóstico dos casos das LEP, vários autores apresentaram classificações sendo a de Simon et al (1972), conforme citado por Cardoso e Miranda (2018), a mais aplicada por se basear nos fatores etiológicos e na forma de disseminação pelas vias anatômicas, além do tratamento e prognóstico. Esta compreende em lesões primariamente endodônticas, lesões primariamente endodônticas com envolvimento periodontal secundário, lesões primariamente periodontais, lesões primariamente periodontais com envolvimento endodôntico secundário e lesões combinadas verdadeiras.

Nas lesões primariamente endodônticas ocorre a perda dos tecidos de suporte periodontais em razão da drenagem da polpa necrótica por meio do forame apical, canal lateral ou até mesmo dos canais acessórios presentes na área de furca. Apesar de dolorosa, em geral não é observada radiograficamente. Quando este tipo de lesão evolui de forma negativa, passamos para presença de uma lesão periodontal verdadeira de natureza crônica, classificada como primariamente endodônticas com envolvimento periodontal secundário (PEERAN et al, 2013). 
A polpa ainda encontra-se vital nas lesões primariamente periodontais, a presença de dor é mínima ou inexistente e normalmente há presença de bolsa periodontal (PEERAN et al, 2013). Caso a lesão periodontal crônica comprometa o fornecimento de sangue para a polpa e com isso ocorra degeneração pulpar, a lesão passa a ser primariamente periodontal com envolvimento endodôntico secundário. As lesões combinadas verdadeiras se caracterizam pela presença de inflamações ou infecções independentes nos tecidos pulpares e periodontais na fase inicial seguida pela união por entre as vias de comunicação (PINTO, 2018).

De outro modo, outra classificação amplamente aplicada é de Weine (1995, apud MAFRA, 2014, p.33) fundamentada na origem da doença, para determinação do tratamento necessário e os possíveis prognósticos, dividindo as lesões endoperio em quatro classes distintas. São essas: Classe I, onde sintomas clínicos e radiográficos sugerem patologia periodontal, mas efetivamente provocada por inflamação pulpar; Classe II, dentes em totalidade com doença periodontal conjunta de doença pulpar ou periapical; Classe III, dentes com doença periodontal, sem lesão pulpar, porém necessitam de tratamento endodôntico associado; e Classe IV, onde sintomas clínicos e radiográficos sugerem patologia endodôntica, mas na verdade sofrem apenas de doença periodontal.

Mafra (2014) corroborando com pensamento de Guldener and Langeland (1982) cita a classificação fundamentada na inter-relação patológica, dividida em: lesão endo-perio, a infecção de origem pulpar escoa para a cavidade bucal através dos tecidos periodontais, podendo ser por meio do ligamento periodontal, osso alveolar, canais acessórios especialmente em área furcal, gerando bolsa periodontal profunda clinicamente observada até o ápice dental; lesão perio-endo, a exposição radicular torna a região susceptível a infecções bacterianas, e através do forâme apical essas bactérias penetram a polpa, resultando em necrose pulpar, ou pulpite crônica caso os microrganismos se estabeleçam nos túbulos dentinários; e por fim, lesão combinada, onde o elemento dental possui focos de infecção no tecido pulpar e ao mesmo tempo, no tecido periodontal porém de maneira separada.

Conforme a linha de classificação em apenas três classes citada acima, Foce (2011) propôs uma nova classificação conforme citado por Bonaccorso e Tripe (2014) sendo: Classe 1, lesões induzidas por placa bacteriana de forma coroa/ápice; Classe 2, lesões periodontais com origem pulpar advindas do forame apical; Classe 3, combinação entre lesões periodontais verdadeiras e lesões endodônticas. $\mathrm{O}$ autor ainda sugere a Classe 4, como uma opção provisória para casos onde os exames clínicos e radiológicos não são conclusivos sobre a origem da lesão com finalidade de facilitar o diagnóstico.

Baseada nos fatores morfológicos e patogênicos também propuseram os pesquisadores Dietrich (2002, apud Medeiros e Bonesso, 2018, p.21) a seguinte classificação: Classe I, lesão apico marginal, podendo ser puramente periodontal, combinado endodôntico-periodontal ou puramente endodôntica; Classe II, lesão periodontal com origem puramente endodôntica, com fistula na margem gengival e profundidade de sondagem normal, variando conforme a existência de ponte óssea, ponte óssea acima de defeito após cirurgia e defeito apical com deiscência óssea.

A seguir aborda-se no próximo capitulo a respeitos das diversas técnicas para o correto diagnóstico das lesões endoperiapicais, desde as mais comumente utilizadas, até as novas opções em estudo, possibilitando assim a escolha do tratamento ideal por parte do cirurgião-dentista.

O diagnóstico preciso é primordial para o tratamento das LEP, que deve ser obtido através de uma anamnese eficiente junto das técnicas odontológicas frequentemente utilizadas, como por exemplo: exame radiográfico, exame visual, sondagem periodontal, testes de vitalidade pulpar, palpação, percussão e mobilidade, rastreamento de fístulas, exposição cirúrgica. Deve-se utilizar da variedade de testes para o diagnóstico diferencial, visto que apenas um teste não é satisfatório para se obter resultados conclusivos. (CASTRO et al, 2011).

Um bom exame clínico é realizado de forma estruturada e completa, composto por uma anamnese detalhada, seguida de exames físicos intra e extraorais. A anamnese é o primeiro passo no diagnóstico das lesões endoperiodontais, onde o cirurgião-dentista investiga através de relatos do paciente o tipo de dor, a origem e evolução da doença. Além disso, neste momento o profissional estabelece um vínculo de confiança com o paciente, e ademais, a melhora da adesão ao tratamento (BRANDÃO et al, 2018).

O exame visual, a sondagem e os testes de vitalidade pulpar complementam a anamnese em relação a origem e classificação, por meio de possíveis achados relacionados a necrose pulpar ou periodontal. Fatores como inflamação, exsudação, presença de fístulas, restaurações extensas, trincas dentais, presença de cálculo, biofilme, gengivite, periodontite são essenciais para essa diferenciação (BASÍLIO et al, 2020).

Através desses exames são descartadas possibilidades de diagnóstico das lesões endoperiodontais de acordo com os resultados, até alcançar a classificação mais adequada possível para lesão em questão. No caso de positivo para o teste de vitalidade pulpar, por exemplo, descarta-se a origem endodôntica e seguido de uma boa sondagem, dar-se-á o diagnóstico a partir da presença ou não de bolsas periodontais, lesões de furca, inflamações nos tecidos periodontais (PAROLIA et al, 2013).

Os testes de vitalidade pulpar e a sondagem são, portanto, essenciais para o diagnóstico diferencial e facilitam na classificação. No caso de respostas positivas aos testes térmicos, deve-se considerar em alguns casos a existência de falsos-positivos, sobretudo em dentes multirradiculares onde a degeneração pulpar apresenta diferentes estágios nos canais radiculares. As sondagens podem ser utilizadas tanto para o diagnóstico, rastreamentos de fístulas cervicais na região do ligamento periodontal, quanto para o prognóstico. A presença de uma única bolsa periodontal sem doença periodontal associada sugere a existência de fratura radicular vertical ou origem endodôntica da lesão (CASTRO et al 2011; BASÍLIO et al 2020).

Souza et al (2016) recomenda a execução de diferentes métodos de testes, particularmente em casos duvidosos, para reduzir assim a dificuldade de interpretação devido a possibilidade de falsos-positivos ou falsos- 
negativos. Além disso, é imprescindível a comparação em dentes vizinhos e homólogos nos testes térmicos e de percussão vertical e horizontal, para se obter um parâmetro das alterações. Castro et al (2011) cita métodos não invasivos que podem auxiliar no diagnóstico, porém ainda sem comprovação científica de resultados significativos em relação as lesões endoperio, nos quais utilizam de aparelhos Doopler, ressonância magnética e oxímetros de pulso.

Marques et al (2020) propôs a utilização da tomografia computadorizada de feixe cônico (TCCB) para o diagnóstico e acompanhamento de lesões endoperio de forma segura, visto que as radiografias intraorais convencionais possuem a limitação de imagens bidimensionais. No entanto, as radiografias periapicais ainda são o método mais utilizado pelos cirurgiões-dentistas pela facilidade no acesso e o baixo custo comparado às TCCB. Esta permite a avaliação dos tecidos periodontais e dentais, além de ser utilizada como parte da análise do tratamento escolhido e na preservação (CASTRO et al, 2011).

As radiografias de lesões em estágio inicial possuem fácil diferenciação em boa parte dos casos, principalmente quando baseia-se na classificação de Simon para compreender a patogênese. Com isso, entende-se a origem da lesão, de forma a ser dividida em primariamente endodôntica com ou sem comprometimento periapical secundário, primariamente periapical com ou sem comprometimento endodôntico secundário, ou combinadas verdadeiras. O diagnóstico é mais desafiador quando as lesões progridem até seu estágio final, pois o aspecto clínico e o aspecto radiográfico estarão semelhantes em todas as classificações (MAFRA, 2014).

Para o diagnóstico diferencial de lesões pulpares, deve-se considerar a história clínica do paciente, sobretudo procedimentos que podem evoluir para necrose pulpar, como restaurações profundas, infiltrações, traumas, desgastes dentários severos. Comumente há dor aguda latejante, edemas localizados, tumefações que estendem-se para os planos faciais, com vias de drenagem pela mucosa, gengiva e sulco gengival. Perda óssea situada nas regiões de crista e furca, e mobilidade dentária apenas em estágios agudos. (PINTO, 2018).

De acordo com Mafra (2014), as lesões endodônticas apresentam resultados negativos em relação aos testes de vitalidade pulpar, ou pouco claros. Em casos de dentes multirradiculares, pode-se realizar um teste de cavidade para maior esclarecimento devido a necrose parcial. Na sondagem periodontal, apresentam apenas um ponto positivo, sendo normalmente um canal fistuloso em forma de "U", estreito e tortuoso, na porção coronal.

Assim como abordado previamente no capítulo um, as lesões endodônticas primárias são dolorosas, com presença de edemas, resultantes de infecções cariosas, traumas ou infiltrações que progridem para necrose pulpar. Há presença de fístula, com drenagem através do ligamento periodontal exteriorizando no sulco gengival, pequena perda de tecidos de suporte, e ocasionalmente, pseudo bolsa periodontal. Pode-se utilizar da técnica de rastreamento com guta-percha para chegar à origem inflamatória, sendo normalmente o ápice ou canais laterais. A sondagem periodontal é mínima, e o exame radiográfico mostra comprometimento localizado, frequentemente na porção apical. (BASILIO, 2020).
A evolução da drenagem via sulco gengival das lesões anteriores, resulta no acúmulo contínuo de placa bacteriana e cálculo, que podem ser observados durante exame clínico com sonda periodontal. Com isso, ocorre maior perda de tecidos de suporte, formação de bolsas periodontais e instalase uma lesão periodontal verdadeira, dessa forma identificase uma lesão primariamente endodôntica com envolvimento periodontal secundário. Radiograficamente apresenta além do comprometimento apical, alterações por toda extensão radicular (CAMARGO, 2017).

Para Pinto (2018), o diagnóstico diferencial de origem periodontal normalmente inicia-se após ao teste de vitalidade pulpar dar positivo, posto que a ausência de polpa vital mostra-se apenas nos casos mais avançados. A sintomatologia dolorosa nem sempre presente, por vezes maçante e crônica, com presença de edemas generalizados limitados a mucosa aderida, sendo usualmente utilizado o teste de percussão para identificar o dente causador. Há mobilidade dentária e perda óssea generalizada, trajeto fistuloso de fácil sondagem, com largura predominante na região cervical, levando a região média da raiz. Radiograficamente a perda óssea ocorre da crista para o ápice.

No contexto de destruição severa e localizada de tecidos periodontais, deve-se considerar a existência de periodontite retrógrada, fenômeno resultante de efeitos deletérios da doença pulpar ocasionando exsudatos inflamatórios purulentos, formação de bolsas periodontais, mobilidade dentária aumentada, perda óssea. Por outro lado, o oposto equivalente seria a pulpite retrógrada (PEERAN et al, 2013; PINTO, 2018).

As lesões periodontais primárias podem ser similares as lesões endodônticas durante os exames clínicos, exceto pela razão da polpa responder positivamente aos testes de vitalidade. Entretanto, devido à alta perda de tecidos de suporte verificada radiograficamente, deve-se atentar para sensibilidade alterada quanto a variações de temperatura. A doença periodontal pode ser observada em outras áreas da cavidade oral, durante a sondagem nota-se bolsa periodontal acentuada com sangramento. Abcessos periodontais são recorrentes, com escoamento do exsudato purulento via sulco gengival (CAMARGO, 2017).

A ausência de tratamento da bolsa periodontal faz com que a infecção siga o curso apical, e com isso agentes contaminantes infiltram o tecido pulpar por meio dos canais laterais ou do forame apical, tornando a polpa necrótica. Com isso, passa-se para o diagnóstico diferencial de lesão primariamente periodontal com envolvimento endodôntico secundário. Durante exame de palpação observa-se reação dolorosa, presença de bolsas profundas através da sondagem e ampla doença periodontal em toda cavidade bucal (SHENOY; SHENOY, 2010).

Os processos endodônticos e periodontais citados anteriormente podem ocorrer de forma simultânea, desenvolvendo-se de formas separadas ou unindo-se em uma única lesão, e com isso dar-se-á o diagnóstico de lesão combinada verdadeira. Testes pulpares são negativos, na sondagem observa-se bolsas periodontais profundas, testes de mobilidade positivos pois há perda de inserção significativa e inflamação perirradicular, sintomatologia dolorosa durante palpação. Nos exames de imagem nota-se lesão apical com 
defeito ósseo irregular de modo vertical ao longo eixo do dente. O nível de destruição periodontal será determinante para o prognóstico (BASÍLIO, 2020).

Considerando as formas de diagnóstico diferencial baseados nas classificações das lesões endoperio abordadas neste capitulo, destaca-se a seguir os possíveis tratamentos de eleição para o melhor prognóstico possível para o paciente.

As lesões endoperiodontais são complexas devido à dificuldade de diagnóstico encontrada em boa parte dos casos, contudo, utilizando-se das diversas ferramentas disponíveis para correta diferenciação, associada a observação detalhada das particularidades do caso em questão, o nível de conhecimento teórico e vivência do profissional, obtém-se por consequência, a correta seleção da sequência terapêutica (CAMARGO,2017).

Para Basílio et al. (2020) os tratamentos convencionais variam em tratamento endodôntico, curetagem da lesão apical, profilaxia e raspagem supra e subgengival, e em alguns casos se faz necessária a escolha de terapêuticas alternativas, como as cirurgias paraendodônticas e ressecções radiculares. A eleição deve ser sempre baseada no diagnóstico, porém nem sempre é possível a definição de um tratamento definitivo. Fagundes et al. (2007) corrobora com a ideia de que em certas ocasiões deve-se optar por um tratamento empírico, com a realização da terapia endodôntica seguida de um período de observação clínica e radiográfica, para então realizar a terapia periodontal.

Não obstante, há estudos que recomendam o tratamento básico periodontal anterior ao tratamento endodôntico, e por fim apenas se necessário, procedimentos periodontais mais complexos, como os cirúrgicos. A literatura dos diversos métodos curativos das LEP nem sempre aborda os mesmos tipos de intervenção, contudo entra em consenso a respeito da importância do diagnóstico diferencial para melhor prognóstico possível, em associação a interdisciplinaridade entre a periodontia e endodontia (SOUZA et al, 2016).

De acordo com Alves (2018), constantemente dentes acometidos por lesões endoperio são condenados à extração devido à falta de conhecimento a respeito do assunto por parte dos cirurgiões-dentistas. Os levantamentos de dados do autor em questão, apontam que a formação da sequência terapêutica envolvendo primariamente o tratamento endodôntico e posteriormente o tratamento periodontal, é o mais aceito, fato que pode estar ligado ao potencial do tratamento endodôntico em controlar grande parcela dos acometimentos ósseo das LEP.

O objetivo principal do tratamento é preservar o máximo possível da dentição natural do paciente, desta forma, baseia-se em parâmetros periodontais, endodônticos e restauradores para o diagnóstico do dente e classificação do seu prognóstico, podendo ser bom, questionável ou mau. Em casos de vitalidade pulpar, por exemplo, deve-se considerar fatores como a dificuldade de irritação intensa do periodonto, apesar da polpa estar em processo inflamatório. Ademais, durante a eleição do tratamento, elencar a possibilidade de restaurações, o nível de comprometimento dos tecidos de suporte, se há colaboração do paciente, qualidade de higiene oral, os custos, e analisar as opções a curto e longo prazo (PINTO, 2018).
O tratamento de eleição deve ser o mais simples com o qual se consiga obter sucesso, eliminando por fim os fatores etiológicos responsáveis pela destruição tecidual. Considerando a vitalidade pulpar e a classe e difusão da doença periodontal, além da origem da lesão, pode-se optar por métodos curativos apenas endodônticos ou periodontais, e em casos mais complexos define-se terapêuticas que abrangem o princípio de relação estreita entre ambas as áreas (MAFRA,2014).

Schacher et al (2007) baseou-se na experiência de uma clínica dentária de Frankfurt para criar um protocolo de tratamento para as LEP conforme citado por Pinto (2018), dividido em cinco etapas. A primeira, chamada de fase zero, dedica-se em remover a dor; a segunda, fase um, dura em torno de dois meses e consiste no tratamento endodôntico radical (TER) associado a reeducação do paciente sobre higiene oral e uma avaliação inicial da sua colaboração com o tratamento; a terceira, primeiro follow-up, seis meses após o TER para avaliação da principal causa da lesão; a quarta, segundo follow up, um ano após o TER para averiguar o desenvolvimento do tratamento e se é necessária a extração ou apenas manutenção; e a fase final um ano e meio depois de finalizar o TER.

Em seguida do diagnóstico de uma lesão endodôntica primária, pode-se iniciar a terapêutica com foco no tratamento endodôntico do canal radicular, sendo o resultado dependente apenas do controle dos microrganismos presentes no conduto, com utilização de medicação intracanal associada a obturação eficiente. Necrose pulpar, pulpite reversível e irreversível, periodontite e abcessos apicais agudos ou crônicos possuem alta taxa de cura através do tratamento endodôntico. Deve-se reavaliar o canal após seis meses por meio de radiografias periapicais, e analisar a resposta dos tecidos de sustentação em relação ao tratamento. A resolução da lesão é rápida e com excelente prognóstico se realizado corretamente (SHARMA et al, 2015).

Perante quadros de lesões endodônticas primárias com envolvimento periodontal secundário deve-se avaliar inicialmente o grau de comprometimento dos tecidos periodontais. Com isso, verifica-se se o TER é indicado e em conformidade com a severidade do envolvimento periodontal o prognóstico pode ser bom ou ruim. A conduta mais indicada é, a princípio, realizar o tratamento endodôntico associado a melhora da higiene bucal, e após seis meses reavaliar a necessidade do tratamento periodontal adicional, já que neste período o periodonto passa pelo processo de cicatrização com risco reduzido de contaminação por agentes infectantes (PAROLIA et al, 2013).

Mafra (2014) afirma que nas lesões periodontais primárias o tratamento meramente periodontal é o bastante. Deve-se iniciar pela fase de higienização oral, orientando o paciente a reduzir o acúmulo de placa, e dessa maneira, redução do tártaro. Locais de perda de inserção clínica superior a três milímetros, realizar raspagem e alisamento radicular. Cirurgias periodontais de forma ponderada, e apenas em casos de bolsas muito profundas, visto que durante esses processos pode haver remoção do cemento, exposição de túbulos dentinários e assim, necrose pulpar. O prognóstico depende da adesão do paciente ao tratamento e as técnicas de 
higiene oral, além da agressividade da doença periodontal e a qualidade da terapêutica aplicada.

Casos de lesões primariamente periodontais com envolvimento endodôntico secundário são mais custosos, já que há o comprometimento do forame apical. Recomenda-se a mesma sequência do tratamento das lesões endodônticas primárias com envolvimento periodontal secundário, porém devido a severidade da doença periodontal pode ser necessária a realização de etapa cirúrgica para regeneração óssea suficiente. A terapia regenerativa é uma boa alternativa frente a casos como dentes multirradiculares com defeitos Classe I e II em áreas de furca, principalmente quando aliados ao emprego de plasma rico em plaquetas (PRP) ou de fibrina rica em plaquetas (PRF) para máxima resposta clínica da regeneração tecidual (ALVES, 2018).

As lesões combinadas verdadeiras são as de prognóstico mais sombrio, com baixas taxas de sucesso caso não haja condições para o processo regenerativo, com isso, a terapêutica nesses pacientes é completa, incluindo tratamento endodôntico, periodontal e procedimentos regenerativos. $\mathrm{O}$ cirurgião-dentista deve considerar amplamente os fatores de influência no tratamento como, função dentária, anatomia, restaurabilidade, qualidade do suporte ósseo, anatomia e a colaboração do paciente para então decidir se é viável (MAFRA,2014).

Embora a patogênese variada e a amplitude das técnicas de diagnóstico apresentarem desafios para o cirurgião-dentista, o tratamento das lesões endoperiodontais pode ser bem-sucedido desde que haja a completa compreensão e o conhecimento científico para o estabelecimento de um correto plano de tratamento. A longo prazo, o prognóstico depende não só do tratamento correto a partir de um bom diagnóstico, como do acompanhamento com motivação ao paciente e da gravidade e extensão da lesão inicial (CASTRO et al, 2011; PAROLIA et al, 2013).

\section{Conclusão}

O termo lesão endoperio vem sendo utilizado para casos onde os processos inflamatórios se enquadram tanto nas classificações das lesões endodônticas, quanto nas lesões periodontais. Com isso, assim como abordado no trabalho, diversos autores apresentaram novas classificações a fim de facilitar o diagnóstico diferencial nesse contexto.

Apesar da complexidade das LEP, a escolha de uma sequência terapêutica correta é possível desde que o cirurgiãodentista faça o correto diagnóstico. Para isso, é necessária a completa compreensão e conhecimento científico para realização de uma anamnese detalhada junto a variedade de testes apresentados, visto que a realização de apenas um teste não é suficiente.

Portanto, o diagnóstico diferencial é de extrema importância para que o tratamento de eleição seja o mais simples com o qual se consiga obter sucesso, eliminando por fim os fatores etiológicos responsáveis pela destruição tecidual. A longo prazo, a gravidade e a extensão da lesão inicial e o acompanhamento com motivação do paciente também são essenciais para um bom prognóstico.

\section{Declaração}

Os autores declaram que não existe conflito de interesse.

\section{Referências}

Alves, VF. Lesão Endo-Perio: Como conduzir clinicamente? Monografia (Trabalho de Conclusão de Curso de Especialização). Universidade Federal de Pernambuco. Sete Lagoas, p.20, 2018.

Basílio, GG. et al. Lesões endodôntico-periodontais. Revista de Odontologia Contemporânea - ROC. v. 4 n. 1, 2020.

Brandão, BA. et al. Importância de um exame clínico adequado para o atendimento odontológico. Cadernos de Graduação: Ciências biológicas e de saúde unit. Alagoas, v.5, n. 1, p. 77-88, Novembro/2018.

Bonarccoso, A; TRIPI, TR. Endo-perio lesion: Diagnosis, prognosis and decision-making. ENDO (Long Engl). 2014;8(2):105-127.

Camargo, RF. Lesões Endo-Periodontais. 2017. 17 d. Dissertação (Mestrado em Medicina Dentária. Faculdade Ciências da Saúde, Universidade Fernando Pessoa, Porto, 2017.

Cardoso, RM; Miranda, JM. Tratamento de lesão endo-pério: relato de caso. Odontol. Clín.-Cient., Recife, 17(3) 225 - 227, jul./set., 2018

Castro, ICV. et al. Lesões endoperiodontais: uma visão contemporânea. C\&D-Revista Eletrônica da Fainor, Vitória da Conquista, v.4, n.1, p.73-86, jan./dez. 2011

Fagundes, F. et al. Lesões endoperiodontais - considerações clínicas e microbiológicas. RSBO Revista Sul-Brasileira de Odontologia, vol. 4, núm. 2, pp. 54-60, 2007.

Mafra, S B. Lesões Endo-Perio: Classificação e Diagnóstico. 2014. 72 d. Dissertação. (Mestrado em Medicina Dentária). Faculdade Ciências da Saúde, Universidade Fernando Pessoa, Porto, 2014.

Marques, EF. et al. Regression of low predictability periapical lesion through endodontic treatment in a single session: case report. International Journal of Advanced Engineering Research and Science (IJAERS). Vol-7, Issue-2, Feb/2020.

Medeiros, IB; Bonesso, NR. Lesões endo-perio: o dilema multidisciplinar - uma revisão de literatura. Monografia (Trabalho de Conclusão de Curso). Universidade de Uberaba. Uberaba, p.47, 2018.

Parolia, A. et al. Endo - perio lesion: A dilemma from 19th until 21st century. Journal of Interdisciplinary Dentistry. 3:2$11,2013$. 
Peeran, SW. et al. Endo-Perio Lesions. International Journal of Scientific \& Technology Research, vol 2, issue 5, may/ 2013

Penoni, DC; Andrade, MAC. Lesão endo-periodontal: um relato de caso. Rev Nav Odontol. 43(1)24-29, 2016.

Pinto, JRLG. Lesões endo-perio: a complexidade do diagnóstico. Tese (Mestrado Integrado em Medicina Dentária). Faculdade de Medicina Dentária da Universidade do Porto. Porto, p.26, 2018.

Santos, ACB; Lins, CCSA. Tomada de decisão no diagnóstico e terapêutica das lesões endopério por endodontistas da cidade de maceió-2007. International Journal of Dentistry. Recife, 6(3):80-85, jul / set 2007

Sharma, N. et al. Endo - Perio lesions: A Diagnostic Dilemma. International Journal of Preventive \& Clinical Dental Research. I J Pre Clin Dent Res 2015;2(4):41-44 Oct-Dec.

Shenoy, N; Shenoy, A. Lesões de Endo-perio: Diagnóstico e considerações clínicas. Indian J Dent Res 2010; 21: 579-85

Souza, LC. et al. Lesão endopério: relato de caso. Brazilian Journal of Surgery and Clinical Research - BJSCR. V.15, n.1,p.53-56, jun/ago,2016. 